\title{
The Need for Psychosocial Interventions to Manage the Coronavirus Crisis
}

\author{
Seyyed Mohammad Hossein Javadi (ii) ${ }^{1}$, Moslem Arian ${ }^{2,{ }^{*}}$ and Milad Qorbani-Vanajemi (iD ${ }^{2}$ \\ ${ }^{1}$ Social Work Department, University of Social Welfare and Rehabilitation Sciences, Tehran, Iran \\ ${ }^{2}$ University of Social Welfare and Rehabilitation Sciences, Tehran, Iran \\ "Corresponding author: University of Social Welfare and Rehabilitation Sciences, Tehran, Iran. Tel: +98-9024659903, Email: arianmoslem@yahoo.com \\ Received 2020 March 10; Accepted 2020 March 13.
}

Keywords: Psychosocial Interventions, Coronavirus, Iran

\section{Dear Editor,}

Coronavirus (COVID-19) has crossed the species boundary and reached humans for the third time (1). To date, March 9th, 2020, reports by the Ministry of Health and Medical Education have indicated that more than 6,000 people have been infected with the coronavirus, and more than 190 people have died in Iran. Experts predict that the outbreak will continue to rise (2). Death, lack of equipped hospitals, difficulty performing the duties, the overwhelming sense of suffering in medical staff, and economic and educational challenges are among the consequences of this epidemiological disease. But these are only part of the facts about the coronavirus. This disease has generally resulted in severe anxiety and psychological problems in the societies involved (3) and seemingly in our country people who are infected and even non-infected are highly susceptible to experience physical, psychological, social, and economic issues.

Some of the basic psychosocial issues generated by the disease seem to be: (I) abnormal mourning for the death of loved ones; (II) home quarantine and disruption of daily living;(III) increased sensitivity and obsession about cleaning, and washing, as well as having anxiety, worries, and psychological distresses; (IV) closure of schools, universities, and offices in some areas, as well as social-educationalrecreational and even home-related effects of disrupting these routines; (V)Increased sensitivity and overwhelming condition in certain careers due to controlling and curing the disease, and; (VI) general psychosocial distress in society as well as problems caused by rumors and debilitation of social capital.

If psychosocial interventions are delayed in crisis, people who are involved may suffer from significant psychological damage (4). We believe that the crisis, with all its damages and disadvantages, can also reinforce and boost human beings. Managing a crisis requires discipline, coordination and flexibility as well as attention to all human issues, and utilization of the specialists in various fields.

The authors recommend that mental health professionals, social workers, and crisis intervention specialists make use of diverse and contextual interventions to address the psychosocial issues of the disease in the country. Now in mainland China, online psychosocial interventions are provided by physicians, psychologists, social workers, and psychiatrists to the general public as well as to the hospital staff involved with the disease $(5,6)$. Likewise, we can think of similar protocols in Iran. Since research findings indicate that anxiety is associated with weakness in the immune system, providing various psychosocial-medical interventions and educations can enhance social capital, community safety and psychosocial well-being and thus be decisive in reducing the disease and its adverse effects.

Therefore, the necessity of designing and formulating short-term and online psychosocial interventions and protocols is felt for different groups of society and specialists in other fields ought to access these interventions and protocols. Online access such as social networks, internet, telephone, and the media can be a good platform for presenting these psychosocial protocols. Various startups can also be used to facilitate access for interventions in different groups of people. The target population of these protocols includes hospitalized patients and their families, home and family caregivers and their patients, medical staff of hospitals and their families, and normal population.

Needless to say, a community is cured by its members, and psychosocial experts are expected to play a significant role in managing this crisis. 


\section{Acknowledgments}

We appreciate the University of Social Welfare and Rehabilitation Sciences.

\section{Footnotes}

Authors' Contribution: Moslem Arian designed the study, collected the data, and drafted the manuscript. Dr. Seyyed Mohammad Hossein Javadi provided scientific content. Milad Qorbani-Vanajemi revised the manuscript. All the authors read and approved the final manuscript.

Conflict of Interests: Authors declared no conflict of interest.

Ethical Approval: All procedures performed in the study on human participants were in accordance with the ethical standards of the Institutional and/or National Research Committee, and the 1964 Declaration of Helsinki and its later amendments, or comparable ethical standards.

Funding/Support: This article is a letter to the editor and no financial support was received from any governmental or academic organizations.

\section{References}

1. Perlman S. Another decade, another coronavirus. $N$ Engl J Med. 2020;382(8):760-2. doi: 10.1056/NEJMe2001126. [PubMed: 31978944].

2. Increased number of patients identified to 5823. 2020. Available from: http://behdasht.gov.ir/index.jsp?siteid=1\&fkeyid=\&siteid=1\& pageid $=55373 \&$ newsview $=200392$.

3. Zhang C, Yang L, Liu S, Ma S, Wang Y, Cai Z, et al. Survey of insomnia and related social psychological factors among medical staffs involved with the 2019 novel coronavirus disease outbreak. SSRN. 2020

4. Jiang X, Deng L, Zhu Y, Ji H, Tao L, Liu L, et al. Psychological crisis intervention during the outbreak period of new coronavirus pneumonia from experience in Shanghai. Psychiatry Res. 2020;286:112903. doi: 10.1016/j.psychres.2020.112903. [PubMed: 32146245].

5. Zhang W, Zhao X, Wu W, Zhang J. Recommended psychological crisis intervention response to the 2019 novel coronavirus pneumonia outbreak in China: A model of West China Hospital. Precis Clin Med.2020. doi: 10.1093/pcmedi/pbaa006.

6. Xiao C. A novel approach of consultation on 2019 novel coronavirus (COVID-19)-related psychological and mental problems: Structured letter therapy. Psychiatry Investig. 2020;17(2):175-6. doi: 10.30773/pi.2020.0047. [PubMed: 32093461]. [PubMed Central: PMC7047000] 